\title{
Efectos inmunológicos de la vitamina D en COVID-19
}

Immunological effects of vitamin D on COVID-19

\author{
Rivera $M C^{1}$, Medina $A^{2}$, Vargas J3, Gómez $A M^{3}$, González E ${ }^{3}$
}

${ }^{1}$ Médica Internista y Endocrinóloga, Hospital Universitario Clínica San Rafael, Bogotá, Colombia.

${ }^{2}$ Médica Internista y Endocrinóloga, Hospital San José, Bogotá, Colombia.

${ }^{3}$ Médico Residente de Medicina Interna, Hospital Universitario Clínica San Rafael, Bogotá, Colombia.

Autor de correspondencia: María Claudia Rivera

Correo electrónico: maclau04@hotmail.com

\section{Resumen}

El síndrome respiratorio severo o grave causado por el nuevo coronavirus (SARS-CoV-2) es una enfermedad de gran auge en la actualidad, que se ha propagado vertiginosamente a lo largo de los 5 continentes, siendo Norteamérica y algunos países europeos como Italia, España y Francia los más afectados por esta pandemia. En la actualidad, no se cuenta con una estrategia de prevención o tratamiento que logre mitigar de forma contundente las cifras de infectados y muertos a nivel mundial; sin embargo, se están estudiando alternativas que podrían impactar de forma positiva en el curso de la enfermedad.

El déficit de 25 hidroxivitamina D (250H D) ha mostrado ser un factor independiente de mortalidad por todas las causas, principalmente en enfermedades cardiovasculares y cáncer. La suplementación de esta también se ha asociado a beneficios en la prevención de enfermedades respiratorias; para el caso de la COVID-19, los mecanismos por los cuales la vitamina $\mathrm{D}$ podría ser útil para el tratamiento y la prevención se resumen en la actividad sobre las barreras físicas y la inmunidad natural celular y adaptativa, que disminuye la probabilidad de tormenta de citocinas.

Algunos estudios realizados concluyeron que el pico de la infección por SARS-CoV-2 se presentó durante el invierno, tiempo donde los niveles de $250 H$ D son más bajos; además, estos niveles subóptimos se han relacionado con aumento en la incidencia de complicaciones tales como falla cardíaca, sepsis y progresión a síndrome de dificultad respiratoria del adulto (SDRA) y, por consiguiente, con un aumento en las tasas de mortalidad, lo cual respalda el papel de la vitamina D en la modificación del curso natural de la enfermedad. Sin embargo, también se prendieron las alarmas, ya que se ha demostrado una prevalencia alta de hipovitaminosis D en Bogotá, Villavicencio, Neiva, Barranquilla y Medellín, que podría exponer a la población colombiana a resultados adversos.

La evidencia indica que la suplementación con vitamina D modula y reduce el riesgo de infección por SARS-CoV-2, pero se precisan más estudios para corroborar los efectos benéficos en la población.

Palabras claves: síndrome de dificultad respiratoria aguda (SDRA), enfermedades crónicas, mortalidad, coronavirus, COVID-19, tormenta de citocinas, influenza, observacional, neumonía, prevención, infección del tracto respiratorio, radiación solar, tratamiento, $\mathrm{UVB}$, vitamina D.

\section{Abstract}

The severe respiratory syndrome, caused by the new coronavirus (SARS-CoV-2), is a currently booming disease that has spread rapidly throughout the 5 continents. North America and some European countries such as Italy, Spain and France have been the most affected by this pandemic. Currently, there is no prevention or treatment strategy that can conclusively mitigate the numbers of infected and dead worldwide; however, alternatives are being studied that could positively impact the course of the disease. The deficiency of 25 hydroxy-vitamin D $(250 \mathrm{H}$ D) has shown to be an independent factor in all-cause mortality, mainly in cardiovascular disease and cancer. Supplementation of it has also been associated with benefits in the prevention of respiratory diseases. In the case of COVID-19, the mechanisms by which vitamin D could be useful for treatment and prevention are summarized in activity on physical barriers, and by natural cellular and adaptive immunity, reducing the probability of cytokine storm. Some studies concluded that the peak of SARSCoV-2 infection occurred during the winter, when the levels of $250 \mathrm{H}$ D are lower. In addition, these suboptimal levels have been related to an increase in the incidence of complications such as heart failure, sepsis, and progression to adult respiratory distress syndrome (ARDS), and consequently an increase in mortality rates. This supports the role of vitamin D in modifying the natural course of the disease. However, the alarms are 
also triggered, since a high prevalence of hypovitaminosis D has been demonstrated in Bogotá, Villavicencio, Neiva, Barranquilla and Medellín that could expose the Colombian population to adverse results. The evidence indicates that vitamin D supplementation modulates and reduces the risk of SARS-CoV-2 infection, but more studies are needed to corroborate the beneficial effects in the population.

Keywords: Acute respiratory distress syndrome (ARDS), Chronic diseases, Mortality, Coronavirus, COVID-19, Cytokine storm, Influenza, Observational, Pneumonia, Prevention, Respiratory tract infection, Solar radiation, Treatment, UVB, Vitamin D.

\section{Introducción}

El síndrome respiratorio severo o grave 2 (SARS-CoV-2), causado por el nuevo coronavirus, fue identificado por primera vez a finales de 2019, en la ciudad de Wuhan, provincia de Hubei, China, donde se propagó rápido y posteriormente fue declarado pandemia. El SARS-CoV-2 es un virus ARN perteneciente al grupo de los betacoronavirus, cuyos miembros ya han sido protagonistas de epidemias en 2002 y 2003 en China, así como el MERS en Medio Oriente. Su estructura vírica es similar a la del virus del síndrome de dificultad aguda grave o SARS, incluso comparten el mismo receptor, el de la enzima convertidora de la angiotensina 2 (ECA-2) para entrar a la célula ${ }^{(1)}$.

Según el observatorio de la Universidad de John Hopkins, al momento de esta publicación, el SARS-CoV-2 ha infectado a 3.113.447 personas en el mundo, de las cuales, 218.930 han fallecido. Estados Unidos es la nación más afectada, con 1.011.877 contagiados, seguida por España e Italia. En Colombia, a la fecha, según el Instituto Nacional de Salud (INS), se han reportado 7000 contagiados y 314 fallecidos. Indudablemente, la pandemia por COVID-19 ha puesto en jaque a los sistemas de salud y a las economías a nivel mundial, por lo que requiere, con premura, tratamientos y estrategias de prevención efectivas ${ }^{(1)}$.

A lo largo de la historia, se ha identificado que el déficit de 25 hidroxivitamina D (250H D) se ha establecido como un factor de riesgo para mortalidad por todas las causas, pero, en especial, por patologías cardiovasculares y cáncer ${ }^{(2)}$. Adicionalmente, también se ha propuesto un beneficio de la suplementación de vitamina $\mathrm{D}$ en la reducción de las tasas de infecciones respiratorias altas, principalmente de influenza, debido a la inducción de la síntesis de defensinas y catelicidinas, además de una modulación de las concentraciones de citocinas proinflamatorias. En cuanto a la COVID-19, teniendo en cuenta que varios estudios informaron que la enfermedad se presentó y se propagó durante las épocas de invierno, tiempo durante el cual los niveles de $250 \mathrm{H}$ D son más bajos en la población general y que el número de personas afectadas fue menor en el hemisferio sur cerca del final del verano, y que, además, varios de los países más afectados, como Italia, Fran- cia y España, tienen una alta tasa de personas con déficit de vitamina D y adultas mayores, lo cual se asoció a una mayor mortalidad frente a otros países europeos, como Alemania, Reino Unido y Suiza, se consideró que la suplementación de vitamina D podría ser útil para el tratamiento y la prevención en pacientes con COVID-19 ${ }^{(3,4)}$.

\section{Materiales y métodos}

Se realizó una búsqueda sistemática en bases de datos como PubMed y Cochrane, donde se logró ubicar artículos sobre la vitamina $\mathrm{D}$, publicados en los últimos 8 años. Como herramienta se utilizaron los términos médicos MeSH de alta frecuencia y, de esta manera, mediante la revisión de cada uno, se logró construir una revisión en relación con los efectos del déficit de la vitamina D con el incremento de la mortalidad, el desarrollo versus prevención de enfermedades de curso crónico y su efecto en el escenario de neumonía de etiología viral, principalmente SARS.

Los términos utilizados fuero: "Acute respiratory distress syndrome (ARDS)"; "chronic diseases"; "Mortality"; "coronavirus"; "COVID-19"; "cytokine storm"; "influenza”; "observational"; "pneumonia"; "prevention"; "respiratory tract infection"; "solar radiation"; "treatment"; "UVB"; "vitamin D".

\section{Evidencia}

Se han encontrado por lo menos 3 mecanismos por los cuales la vitamina D disminuye el curso de infecciones y muerte, como son: barrera física, inmunidad natural celular e inmunidad adaptativa. En cuanto a su efecto sobre la barrera física, la vitamina D incrementa las cadherinas, que son glicoproteínas transmembrana responsables de las uniones célula-célula para mantener la integridad de los tejidos animales, evitando que los virus alteren la integridad de la unión. En su papel sobre la inmunidad celular, estudios han demostrado que diversas células del sistema inmunitario expresan el receptor de vitamina D (VDR), por el cual la vitamina D modula la respuesta inmunitaria, formando un heterodímero con el receptor retinoide $\mathrm{X}$, que, al translocarse al núcleo, se une a elementos de respuesta específicos en el ADN y regula la transcripción de genes en las células diana ${ }^{(3,5-7)}$. Esta acción inmunomoduladora de la vitamina D resulta en potenciación de la respuesta inmunitaria innata, particularmente monocitos y macrófagos, asociada a disminución en la respuesta inmunitaria adaptativa ${ }^{(3,5,7)}$.

Adicionalmente, se ha visto un incremento en la producción de citocinas antiinflamatorias, como la interleucina 10 (IL-10) y el factor de crecimiento transformante beta (TGF- $\beta$ ), asociado a una disminución de las citocinas proinflamatorias, como la IL-17A, IL-17F, IL-22, IL-23, IL-12, IL-2, IL-6, factor de necrosis tumoral alfa (FNT- $\alpha$ ) e interferón (IFN) ${ }^{(3,8,9)}$.

La modulación del sistema adaptativo innato está dada por la activación de los receptores tipo Toll (TLR) presentes en ma- 
crófagos, polimorfonucleares, monocitos y células epiteliales de la conjuntiva, la córnea, el tracto respiratorio y el tracto digestivo, que llevan a la expresión incrementada de VDR y activación de la vitamina D por la enzima 1 alfa hidroxilasa. Cuando los TLR entran en contacto con antígenos como los lipopolisacáridos, los ácidos nucleicos o las proteínas virales, liberan citocinas que inducen a la formación de péptidos antimicrobianos, catelicidinas y defensinas, mediante la vitamina D activa ${ }^{(3,9)}$.

Se ha demostrado la capacidad de las catelicidinas contra virus, bacterias e incluso hongos, al parecer con un efecto directo sobre la membrana de estos organismos, así como también causando neutralización de las endotoxinas ${ }^{(10)}$.

En cuanto a las sustancias evaluadas en la regulación de la vitamina D, están la catelicidina humana y su interacción con el VDR, que ha demostrado tener efectos antivirales por la inhibición del virus herpes simple tipo 1 y en la replicación de algunos serotipos de adenovirus. El aumento de otras moléculas gracias a la acción de la vitamina $\mathrm{D}$, como la defensina $\beta_{2}$, ejerce un efecto antiviral, ya que esta actúa como quimioatrayente de neutrófilos y monocitos ${ }^{(3,9)}$.

El SARS-CoV-2 se une a las células hospederas del ser humano a nivel pulmonar, a través del receptor de ECA-II; a su vez, la vitamina D disminuye la actividad del sistema reninaangiotensina-aldosterona a través de la inhibición de la cascada de renina/ECA/AngII/AT1R. Es así como la vitamina D podría ser un factor contribuyente en la disminución de la aparición de lesión pulmonar aguda (LPA) y síndrome de dificultad respiratoria aguda (SDRA). Los altos niveles de expresión de ECA/Ang II, así como la disminución de la expresión de ECA-2/Ang, están involucrados en la LPA (Figura 1), de tal forma que los niveles de expresión aumentados de ECA-2 y el receptor de vitamina $\mathrm{D}$ (VDR) desempeñan un papel protector contra el desarrollo de SDRA ${ }^{(11)}$.

\section{Discusión}

Numerosas revisiones señalan las características por las cuales la vitamina D reduce el riesgo de infecciones virales (9, 12-15). La vitamina D disminuye la producción de citocinas proinflamatorias, como el IFN y el FNT ${ }^{(3,9)}$, las cuales han sido encontradas elevadas en pacientes infectados con COVID-19 $(13,16-18)$. Adicionalmente, los estudios realizados en los virus SARS-CoV y MERS-CoV evidenciaron que la tormenta de citocinas proinflamatorias lleva a desenlaces graves de la infección, como el SDRA ${ }^{(19,20)}$.

Estudios realizados con el virus de la influenza han señalado un pico de infección durante el invierno ${ }^{(15)}$, lo cual podría estar relacionado con una disminución en la cantidad de rayos ultravioleta recibidos y, por consiguiente, con dosis más bajas de vitamina D. ${ }^{(15,16)}$. Un estudio aleatorizado, realizado en el año 2010 en 162 estudiantes japoneses, obtuvo resultados en los que sugiere que la suplementación con vitamina D, durante la época invernal, puede reducir la incidencia de influenza $\mathrm{A}^{(16)}$.
Figura 1. Deficiencia de la vitamina D y su relación con los principales determinantes de mortalidad en la COVID-19 (11).

Ang: angiotensina; Ang II: angiotensina II; ECA: enzima convertidora de la angiotensina; ECA-2: enzima convertidora de la angiotensina 2; HTA: hipertensión; SDRA: síndrome de dificultad respiratoria aguda.

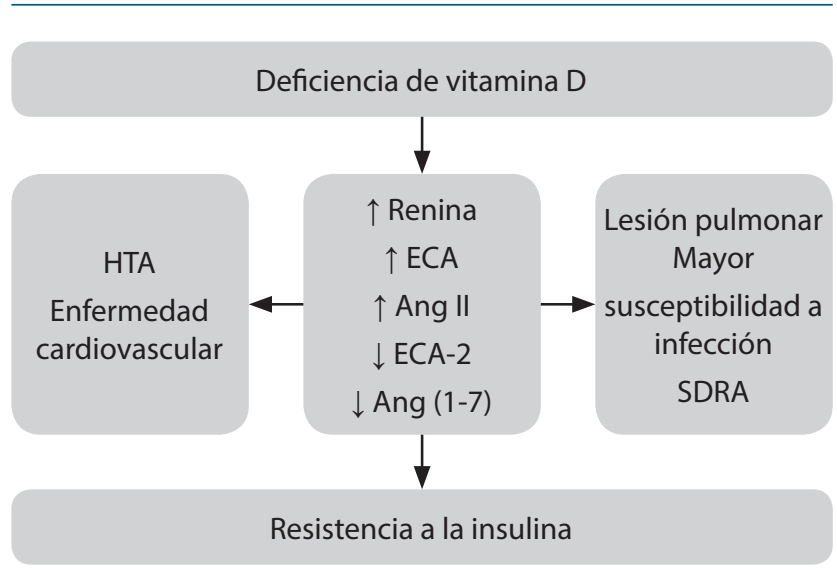

En Italia, que cuenta con una alta población mayor de 70 años, la tasa de mortalidad es la más alta en Europa, con un $11,9 \%$. En las latitudes del norte, la mortalidad está entre el 5,2\% y el 22\%; en el Ecuador o el trópico, entre el 3,1\% y el 9,5\%; y en las latitudes del sur, entre el 0,7\% y el 8,7\%. Estas cifras apoyarían la tesis de la vitamina D como factor determinante de gravedad ${ }^{(17)}$.

En el año 2018, una revisión de la literatura sobre los efectos de la vitamina D en la influenza señaló, luego de evaluar múltiples estudios aleatorizados controlados, que aunque se requieren más estudios para afirmar la relación benéfica de la vitamina D en la infección por el virus de la influenza, es indudable que esta vitamina tiene un efecto modulador en la respuesta inmunitaria, por lo cual el mantenimiento de niveles séricos óptimos debería considerarse para la prevención de enfermedades ${ }^{\left({ }^{8}\right)}$. Dada la variabilidad de la prueba, se consideran como niveles óptimos entre 30 y $40 \mathrm{ng} / \mathrm{dL}$.

Esta información respalda el papel de la vitamina D en la reducción del riesgo de presentar COVID-19 y en el progreso de la enfermedad hacia desenlaces graves; también se ha evidenciado el pico de infecciones cuando los niveles de vitamina D están bajos, lo que sugiere que la suplementación de vitamina D podría prevenir y retrasar el curso natural de la COVID-19. También puede ser una relación circunstancial, en la que los individuos con patologías asociadas a mortalidad por COVID-19, como lo son los cardiópatas, los hipertensos, los obesos, los ancianos y los diabéticos, presentan niveles séricos de $250 \mathrm{H}$ D usualmente bajos ( $<20 \mathrm{ng} / \mathrm{dL}$ ).

El Consenso Colombiano de Vitamina D reportó en el año 2017 una alta prevalencia de hipovitaminosis D en ciudades como Bogotá, Villavicencio, Neiva, Barranquilla y Medellín ${ }^{(19)}$, lo cual, teniendo en cuenta la información analizada y los estudios 
encontrados, podría predisponer a dicha población colombiana a contraer con mayor facilidad el virus COVID-19 y a presentar mayores desenlaces graves. Según argumentan diversas revisiones y estudios ${ }^{(8,15,16,22)}$, y a pesar de que los resultados no han sido concluyentes, la evidencia indica que la suplementación de vitamina D modula y reduce el riesgo y la gravedad de la infección; sin embargo, es necesario realizar más estudios en diversas poblaciones para corroborar los beneficios de la suplementación de vitamina D contra la infección.

Este mismo consenso de 2017 también determinó $30 \mathrm{ng} /$ $\mathrm{mL}$ como el nivel de concentración sérica adecuado de $250 \mathrm{H}$ ${ }^{(21)}$; sin embargo, en otros estudios relacionados con la prevención de infección en el tracto gastrointestinal, se describen como niveles séricos deseables entre 40 y $60 \mathrm{ng} / \mathrm{dL}^{(19,23)}$.

La fortificación de vitamina D mediante la ingesta de alimentos en productos lácteos y harinas podría aumentar sus niveles ${ }^{(20)}$, lo que sería muy valioso en aquellas poblaciones de bajo nivel socioeconómico y con bajos niveles séricos de vitamina D en Colombia, así como la exposición solar responsable, la cual está disminuida por el aislamiento preventivo; no obstante, se recomienda la suplementación diaria o semanal de vitamina D para obtener mejores resultados ${ }^{(24)}$.

La dosis que se recomienda en el contexto de la epidemia por COVID-19 es muy variable, dado que existen estudios con dosis de 2000 UI diarias en pacientes con déficit de vitamina, que ha demostrado ser suficiente para prevenir el desarrollo de las infecciones del tracto respiratorio ${ }^{(25,26)}$. Por otra parte, un estudio realizado en pacientes críticamente enfermos con soporte ventilatorio habla de dosis altas de 250.000 o 500.000 UI de carga (en promedio, de 50.000 UI o 100.000 UI/d, por 5 días). Estos pacientes, además del déficit de vitamina $\mathrm{D}$, tenían anemia, y en aquellos con dosis más altas de vitamina $\mathrm{D}$ disminuyeron las concentraciones de hepcidina, que por su papel a nivel hepático en el metabolismo del hierro mejoró de los niveles de hemoglobina y la capacidad de la sangre para transportar oxígeno ${ }^{(26)}$. Pese a esto, un aspecto importante es la intoxicación por vitamina $\mathrm{D}$, que, si bien es muy rara, debe evitarse. Hasta ahora, no hay un consenso en cuanto a la administración de cargas altas de vitamina $\mathrm{D}$, ni de llevar niveles séricos a más de $60 \mathrm{ng} / \mathrm{dL}$. Para la prevención de las infecciones respiratorias por SARS e influenza, se requieren dosis de 2000 a $5000 \mathrm{U}$ diarias, sin necesidad de medir los niveles séricos de vitamina D. Esta dosis es recomendada principalmente en épocas de epidemia o brote respiratorio y en épocas de invierno e incluye a la comunidad en general y al personal de salud; la prevención de este tipo de infecciones se asocia a niveles séricos de vitamina D entre 40 y $60 \mathrm{ng} / \mathrm{dL}^{(15)}$.
En términos generales, la Endocrine Society recomienda una dosis de 1000-4000 UI/24 horas de vitamina D para mantener niveles séricos de $250 \mathrm{H}$ D > $20 \mathrm{ng} / \mathrm{dL}^{(27)}$.

\section{Conclusiones}

Una forma en que el virus SARS-CoV-2 daña a las células epiteliales del pulmón y causa neumonía es mediante el aumento en la producción de citocinas proinflamatorias como parte de la respuesta inmunitaria innata a las infecciones virales, lo que da lugar a la tormenta de citocinas, respuesta inflamatoria exagerada, que en conjunto con unos niveles de vitamina $\mathrm{D}$ en rango de déficit, explicados por la época invernal en la que se presentan los picos de las infecciones respiratorias, facilitan el desarrollo de complicaciones agudas, exacerbación de patologías de curso crónico e incremento en la mortalidad.

El papel de la vitamina D en el sistema inmunitario es bien conocido por su efecto sobre la inmunidad celular, la inmunidad adaptativa y sobre la ECA y ECA-2, que favorece una modulación inmunológica que previene la gravedad de las manifestaciones respiratorias de la COVID-19.

Teniendo en cuenta lo anterior y basados en la evidencia, se podría establecer que el hecho de administrar vitamina D en los meses previos al inicio de la temporada invernal tendría un efecto profiláctico y protector frente al desarrollo de infecciones respiratorias agudas no solamente ocasionadas por CoV, sino también por influenza y las de etiología bacteriana, así como de sus complicaciones. La suplementación de vitamina D puede prevenir la infección por virus SARS y, sobre todo, las manifestaciones graves. La dosis es variable y puede ir de 2000 a $5000 \mathrm{UI} / \mathrm{d}$, sin una fuerte evidencia para el uso de cargas, excepto en pacientes críticos.

Finalmente, en el momento en que nos encontramos de pandemia por COVID-19, se hace urgente una investigación que pueda establecer la relación directa y no circunstancial de la gravedad de esta infección con los niveles de vitamina D y la dosis específica para lograr los efectos inmunomoduladores en infección por SARS-CoV- 2.

\section{Conflictos de interés}

Los autores declaran no tener ningún tipo de conflicto de interés en este tema.

\section{Financiación}

No se recibió ningún tipo de financiación para la realización de este artículo. 


\section{Referencias}

1. Grupo ACIN-IETS de Consenso Colombiano para recomendaciones de atención COVID19. Consenso colombiano de atención, diagnóstico y manejo de la infección por SARS-COV-2/COVID-19 en establecimientos de atención de la salud. Recomendaciones basadas en consenso de expertos e informadas en la evidencia. Infectio. 2020;24(3):1-153. doi: 10.22354/in.v24i3.851.

2. Chowdhury R, Kunutsor S, Vitezova A, Oliver-Williams C, Chowdhury S, Kiefte-de-Jong J, et al. Vitamin D and risk of cause specific death: systematic review and meta-analysis of observational cohort and randomised intervention studies. BMJ. 2014;348:g1903. doi: 10.1136/bmj.g1903.

3. Sassi F, Tamone C, D'Amelio P. Vitamin D: Nutrient, Hormone, and Immunomodulator. Nutrients. 2018;10(11):1656. doi:10.3390/nu10111656.

4. Daneshkhah A, Eshein A, Subramanian H, Roy HK, Backman V. The Possible Role of Vitamin D in Suppressing Cytokine Storm and Associated Mortality in COVID-19 Patients. medRxiv. 2020. doi: 10.1101/2020.04.08.20058578.

5. Teymoori-Rad M, Shokri F, Salimi V, Marashi SM. The interplay between vitamin D and viral infections. Rev Med Virol. 2019;29(2):e2032. doi:10.1002/ rmv.2032.

6. Beard JA, Bearden A, Striker R. Vitamin D and the anti-viral state. J Clin Virol. 2011;50(3):194-200. doi:10.1016/j.jcv.2010.12.006.

7. Greiller CL, Martineau AR. Modulation of the immune response to respiratory viruses by vitamin D. Nutrients. 2015;7(6):4240-70. doi: 10.3390/nu7064240.

8. Gruber-Bzura BM. Vitamin D and Influenza-Prevention or Therapy? Int J Mol Sci. 2018;19(8):2419. doi: 10.3390/ijms 19082419.

9. Korf H, Wenes M, Stijlemans B, Takiishi T, Robert S, Miani M, et al, 1,25-Dihydroxyvitamin D3 curtails the inflammatory and T cell stimulatory capacity of macrophages through an IL-10-dependent mechanism. Immunobiology. 2012;217(12):1292-300. doi: 10.1016/j.imbio.2012.07.018

10. Agier J, Efenberger M, Brzezińska-Błaszczyk E. Cathelicidin impact on inflammatory cells. Cent Eur J Immunol. 2015;40(2):225-35. doi:10.5114/ ceji.2015.51359.

11. Ghavideldarestani M, Honardoost M, Khamseh ME. Role of vitamin D in pathogenesis and severity of COVID-19 infection. Preprints. 2020;202004355. doi:10.20944/preprints202004.0355.v1.

12. Gombart AF, Pierre A, Maggini S. A Review of Micronutrients and the Immune System-Working in Harmony to Reduce the Risk of Infection. Nutrients. 2020;12(1):236. doi: 10.3390/nu12010236.

13. Huang C, Wang Y, Li X, Ren L, Zhao J, Hu Y, et al. Clinical features of patients infected with 2019 novel coronavirus in Wuhan, China. Lancet. 2020;395(10223):497-506. doi: 10.1016/S0140-6736(20)30183-5.

14. Kalil AC, Thomas PG. Influenza virus-related critical illness: pathophysiology and epidemiology. Crit Care. 2019;23(1):258. doi: 10.1186/s13054-019-2539-x.

15. Hope-Simpson RE. The role of season in the epidemiology of influenza. J Hyg (Lond). 1981;86(1):35-47. doi: 10.1017/s0022172400068728.

16. 16. Grant WB, Lahore H, McDonnell SL, Baggerly CA, French CB, Aliano JL, et al. Evidence that Vitamin D Supplementation Could Reduce Risk of
Influenza and COVID-19 Infections and Deaths. Nutrients. 2020;12(4):E988 doi:10.3390/nu12040988

17. Worldometer. COVID-19 coronavirus pandemic [Internet]. Disponible en: https://www.worldometers.info/coronavirus/.

18. Urashima M, Segawa T, Okazaki M, Kurihara M, Wada Y, Ida H. Randomized trial of vitamin D supplementation to prevent seasonal influenza A in schoolchildren. Am J Clin Nutr. 2010;91(5):1255-60. doi: 10.3945/ajen.2009.29094.

19. Quraishi SA, Bittner EA, Blum L, Hutter MM, Camargo CA Jr. Association between preoperative 25-hydroxyvitamin D level and hospital-acquired infections following Roux-en-Y gastric bypass surgery. JAMA Surg. 2014;149(2):112-8. doi: 10.1001/jamasurg.2013.3176.

20. Pilz S, März W, Cashman KD, Kiely ME, Whiting S, Holick MF, et al. Rationale and Plan for Vitamin D Food Fortification: A Review and Guidance Paper Front Endocrinol (Lausanne). 2018;9:373. doi: 10.3389/fendo.2018.00373.

21. Vásquez D, Cano C, Gómez A, González M, Guzmán R, Martínez J, et al. Vitamina D. Consenso Colombiano de Expertos. Medicina. 2017;39(2):140-57.

22. Zhou F, Yu T, Du R, Fan G, Liu Y, Liu Z, et al. Clinical course and risk factors for mortality of adult inpatients with COVID-19 in Wuhan, China: a retrospective cohort study Lancet. 2020;395(10229):1054-62. doi: 10.1016/S01406736(20)30566-3.

23. Laviano E, Sanchez Rubio M, Gonzalez-Nicolás MT, Palacian MP, López J, Gilaberte Y, et al. Association between preoperative levels of 25-hydroxyvitamin $\mathrm{D}$ and hospital-acquired infections after hepatobiliary surgery: A prospective study in a third-level hospital. PLoS One. 2020;15(3):e0230336. doi: 10.1371/ journal.pone. 0230336 .

24. Martineau AR, Jolliffe DA, Greenberg L, Aloia JF, Bergman P, Dubnov Raz G, et al. Vitamin D supplementation to prevent acute respiratory infections: individual participant data meta-analysis. Health Technol Assess. 2019;23(2):1-44. doi: $10.3310 / \mathrm{hta} 23020$

25. Rondanelli M, Miccono A, Lamburghini S, Avanzato I, Riva A, Allegrini P, Faliva MA, et al. Self-Care for Common Colds: The Pivotal Role of Vitamin D, Vitamin C, Zinc, and Echinacea in Three Main Immune Interactive Clusters (Physical Barriers, Innate and Adaptive Immunity) Involved during an Episode of Common Colds-Practical Advice on Dosages and on the Time to Take These Nutrients/Botanicals in order to Prevent or Treat Common Colds. Evid Based Complement Alternat Med. 2018:2018:5813095. doi: 10.1155/2018/5813095.

26. Smith EM, Jones JL, Han JE, Alvarez JA, Sloan JH, Konrad RJ, Zughaier SM, et al. High-Dose Vitamin D3 Administration Is Associated With Increases in Hemoglobin Concentrations in Mechanically Ventilated Critically Ill Adults: A Pilot Double-Blind, Randomized, Placebo-Controlled Trial. JPEN J Parenter Enter Nutr. 2018;42(1):87-94. doi: 10.1177/0148607116678197.

27. Holick MF, Binkley NC, Bischo-Ferrari HA, Gordon CM, Hanley DA, Heaney RP, et al. Evaluation, treatment, and prevention of vitamin D deficiency: An Endocrine Society clinical practice guideline. J Clin Endocrinol Metab. 2011;96(7):1911-30. doi: 10.1210/jc.2011-0385. 